Series A

I. MATHEMATICA

$336 / 7$

\title{
MIT HARMONISCHEN FELDERN VERWANDTE DIFFERENTIALFORMEN UNTER RAND UND ANFANGSBEDINGUNGEN
}

VON

ERNST HÖLDER 
Am 11. Januar 1963 vorgelegt von P. J. Mrrberg und Olli Lehto 


\section{Mit harmonischen Feldern verwandte Differentialformen unter Rand- und Anfangsbedingungen}

Die mathematische Physik kann ihre klassischen Felder (statische Spannungsfelder oder Wellenfelder) in weit grösserem Umfang als harmonisches Feld charakterisieren, wenn dieser Begriff tensoriell erweitert wird und, von der mehrdimensionalen Variationsrechnung geleitet, unabhängig voneinander Übertragung und Metrik eingeführt werden. Ausserdem müssen, wie es bei den harmonischen Feldern Morrey [59] und Friedrichs [14] getan haben, Randbedingungen gestellt werden, bzw. bei den normal hyperbolischen Differentialgleichungen, die sich bei indefinitem Linienelement (etwa der Relativitätstheorie) ergeben, Anfangsbedingungen.

Harmonische Felder vom elliptischen Typ sind bei den bewegungsinvarianten Variationsproblemen z. B. die Selbstspannungen eines mehrfach zusammenhängenden elastischen Körpers.

Ausserdem gibt es noch einen allgemeinen Fall, in dem harmonische Felder mit mehrdeutigen Integralen eine Behandlung versprechen. Man muss für die Matrix der Krümmungsformen $\Omega$ der Übertragung die Gleichung

$$
D^{\prime} \Omega=0
$$

voraussetzen, eventuell auch noch mit Bochner [4]

$$
D \Omega=0 \text {. }
$$

Diese Annahmen bilden eine schwache Brücke zum zweiten Teil des Vortrags ${ }^{1}$ ). Dort untersuche ich harmonische Felder vom hyperbolischen Typ bei indefinitem Riemannschen Linienelement: die elektromagnetischen Felder im leeren Einsteinschen Gravitationsfeld, wo ja auch (0.1), (0.2) gelten. Hier wird die Abhängigkeit von den Anfangswerten studiert: das Huygenssche Prinzip im engeren Sinne Hadamards, also die Diffusion von Licht, die auch im Gravitationsfeld in erster Ordnung des unendlich Kleinen fehlt, aber durchaus vorhanden sein mag, wenn das Licht sich über kosmische Distanzen ausbreitet.

1) Beide Teile wurden im Vortrag gestreift, etwa Nr. 1, 2 und Nr. 3. - Herrn Rolf Nevanlinna danke ich nicht nur für die Einladung dazu, sondern auch für sein Interesse an einer Ausarbeitung, die hier freilich noch skizzenhaft ist und für den zweiten Teil ganz fehlt. 
Nach dem Quantengesetz $\Delta E=h \Delta v$ könnte man vielleicht aus dem Verlust $\Delta E(<0)$ auf eine Frequenzverminderung $\Delta v<0$, eine kosmische Rotverschiebung, schliessen.

1. Harmonische Felder (in der Bezeichnung von Kodaira [43]) auf einer kompakten $\mu$-dimensionalen Mannigfaltigkeit $T$ sind nach Hodge [31], de Rham [64] und Morrey [58]-[60], Friedrichs [14] (die einen Rand $\partial T$ zulassen) überall reguläre Lösungen der linearen partiellen Differentialgleichungen

$$
\begin{aligned}
& D \xi=0, \\
& D^{\prime} \xi=0
\end{aligned}
$$

für die eine Differentialform

$$
\xi=\xi_{\alpha} d t^{\alpha},
$$

wobei die duale Differentialform

$$
* \xi=\sqrt{|g|} g^{\imath \varkappa} \xi_{\varkappa} \varepsilon_{\imath(\alpha)} d t^{\alpha_{1}} \ldots d t^{\alpha_{\mu-1}}
$$

ist,

$$
-* D_{*}=D^{\prime}
$$

( $=\delta=d^{a}$ bei de Rham [64] bzw. Kähler [40]-[41]). Für $\mu=2$ sind es die Abelschen Differentiale 1. Gattung der Funktionentheorie:

$$
\begin{aligned}
\xi & =u d x+v d y, \\
{ }_{*} \xi & =u d y-v d x .
\end{aligned}
$$

Analoge Konforminvarianz gilt, falls die Dimension $\mu=2 p, p$ Grad von $\xi$, ist, s. Nr. 3 .

Wir haben einen richtigen Rand $\partial T$, nicht etwa »offene» Riemannsche Flächen. Wie bei Morrey [59] soll entweder die Tangentialkomponente

$$
t \xi=0 \quad \text { auf } \partial T \quad \text { (Funktionenraum } \mathfrak{F}_{2}^{-} \text {) }
$$

oder die Normalkomponente

$$
n \xi=0 \quad \text { auf } \quad \partial T \quad \text { (Funktionenraum } \mathfrak{P}_{2}^{+} \text {) }
$$

sein.

Mit einem harmonischen Feld "veruandt» nennen wir eine verallgemeinerte vektorwertige Differentialform $\xi^{i}$, vgl. Lichnerowicz [48], Kähler [40]-[41], sowie neuerdings Haahti und Klemola [22],

$$
\xi^{i}=\xi_{\alpha}^{i} d t^{\alpha} \quad(i=1, \ldots, n),
$$


die lokal absolute Ableitung $D x^{i}$ eines Vektors $x^{i}$ ist und durch ein Dirichletsches Prinzip

$$
(D x, D x)=\left(D x^{i}, D x^{i}\right) \rightarrow \min ,
$$

also durch ein quadratisches Variationsprinzip spezieller Art charakterisiert wird.

Ich verwendete in [33], [35], [36] für die 2. Variation eines mehrfachen Extremalintegrals den Cartanschen Kalkül der Differentialformen, den Boerner [5] zuerst für die 1. Variation und geodätische Felder benutzt hat.

In (1.10) gehört das absolute (äussere) Differential

$$
D x^{i}=d x^{i}+\omega_{l}^{i} x^{l}=\xi^{i}=\xi_{\alpha}^{i} d t^{\alpha}
$$

zu einer $\ddot{U}$ bertragung $(D)$, die unabhängig ist von der Metrik $(c)$ des Skalarproduktes,

(1.12) $(\xi, \xi)=\int_{T} \xi \wedge_{c} \xi, \quad$ gleichbedeutend mit $\quad\left(\xi^{i}, \xi^{i}\right)=\int_{T} \xi^{i} \wedge_{c} \xi_{i}$,

mit

$$
{ }_{c} \xi_{i}=(d t)_{\alpha} c_{i j}^{\alpha \beta} \xi_{\beta}^{j}, \quad(d t)_{\alpha}=(-1)^{\alpha-1} d t^{1} \ldots d t^{\alpha-1} d t^{\alpha+1} \ldots d t^{\mu},
$$

wie das auch Bochner [3] ins Auge fasst.

$\mathrm{Zu}$ beachten ist, dass es sich um eine Pseudometrik handelt, die indefinit sein kann. Eine solche indefinite Metrik haben R. Nevanlinna [61] und Louhivaara [52]-[53] behandelt.

Wir machen nämlich nur die Voraussetzung der »starken Elliptizität» (Legendre-Bedingung)

$$
c_{i j}^{\alpha \beta} z^{i} z^{j} y_{\alpha} y_{\beta} \geqq \varepsilon|z|^{2}|y|^{2}, \quad \varepsilon>0 .
$$

Das Variationsproblem (1.10) mit $f(\xi)={ }_{*} f(\xi)(d t),(d t)=d t^{1} \ldots d t^{u}$,

$$
I=\int_{T} f(\xi)=\frac{1}{2}(D x, D x) \rightarrow \min
$$

hat bei der Variation $\delta x=z$ die 1. Variation

$$
\delta I=(\xi, D z)=\left(D^{\prime} \xi, z\right)+\int_{\partial T} \xi z
$$

mit dem adjungierten absoluten äusseren Differential $D^{\prime} \xi_{i}$, das man explizit so berechnet:

$$
\delta I=\int f_{\xi} \delta \xi=\int \pi_{i} \delta \xi^{i}
$$

mit 


$$
\begin{gathered}
f_{\xi^{i}}=f_{\xi_{\alpha}^{i}}(d t)_{\alpha}=\pi_{i}=\pi_{i}^{\alpha}(d t)_{\alpha}\left(=(d t)_{\alpha} c_{i j}^{\beta \alpha} \xi_{\beta}^{j}={ }_{c} \xi_{i}\right), \\
\delta \xi^{i}=d \delta x^{i}+\omega_{l}^{i} \delta x^{l}, \quad \delta \xi=\delta D x=D \delta x=D z,
\end{gathered}
$$

somit

$$
\delta I=-\int_{T}\left(d \pi_{i}-\pi_{l} \wedge \omega_{i}^{l}\right) \delta x^{i}+\int_{\partial T} \pi_{i} \delta x^{i} .
$$

Also gibt

$$
\delta I=0
$$

die Relationen

$$
\begin{aligned}
& D \pi_{i}=d \pi_{i}-\pi_{l} \wedge \omega_{i}^{l}=D_{c} \xi_{i}=0 \text { in } T \\
& t \pi_{i^{\prime}}=0 \quad \text { auf } \partial T \text {, falls } x^{i^{\prime}} \text { frei und } x^{i^{\prime \prime}}=0 \text { auf } \partial T
\end{aligned}
$$

ist. Es kommt also zu

$$
\begin{aligned}
& D x^{i}=\xi^{i}, \\
& D^{\prime} \xi_{i}=0
\end{aligned}
$$

mit $D^{\prime}=-* D_{c}$, wo * den Übergang von der Differentialform $D_{c} \xi_{i}=\left(D_{\alpha} \pi_{i}^{\alpha}\right)(d t)$, mit der kovarianten Ableitung $D_{\alpha}$, zum Skalar $D_{\alpha} \pi_{i}^{\alpha}$ bedeutet.

Übrigens benötigt man für die komplette Theorie der 2. Variation mehrfacher Extremalintegrale noch ein allgemeineres akzessorisches Variationsproblem

$$
\left(D x^{i}, D x^{i}\right)-\left(a_{i j} x^{j}, x^{i}\right) \rightarrow \min
$$

mit der Randbedingung für gewisse $i^{\prime \prime}$

$$
x^{i^{\prime \prime}}=0 \quad \text { auf } \partial T .
$$

Es ist in letzter Zeit vor allem von Hestenes [28] ausführlich für allgemeinere in der Variationsrechnung vorkommende Randbedingungen untersucht worden. Hilbertraummethoden, die er [27] bereits 1951 anwandte, sind auch von van Hove [38], Hölder [35]-[36], Klötzler [42], Hildebrandt [29]-[30] benutzt worden. In den letzten Arbeiten wird die Variationsmethode (bzw. die Methode der orthogonalen Projektion), die Morrey [59]-[60] bei den harmonischen Integralen entwickelt hat, auf die Variationsgleichung übertragen.

Das Eigenwertproblem

$$
\begin{aligned}
& D x^{i}=\xi^{i} \\
& D^{\prime} \xi_{i}=\mu a_{i j} x^{j}
\end{aligned}
$$


ist die Verallgemeinerung des entsprechenden Eigenwertsproblems, das H. A. Schwarz für das Minimum der Minimalflächen in der Festschrift für Weierstrass entwickelt hat - in den Acta Societatis Scientiarum Fennicæ [67].

2. Aber lassen wir den (a)-Term in der 2. Variation und begnügen uns mit einer Verallgemeinerung der harmonischen Felder, also mit Differentialformen $\xi^{i}$, die den Differentialgleichungen (1.23), (1.24) genügen, aber mit einem eventuell mehrdeutigen Vektor $x^{i}$.

Es ist ja im allgemeinen

d. h.

$$
D D x^{i} \neq 0,
$$

neben

$$
D \xi^{i} \neq 0
$$

$$
D^{\prime} \xi_{i}=0,
$$

also nicht $D \xi^{i}=0, \quad D^{\prime} \xi_{i}=0$, was die nächstliegende vektorielle Verallgemeinerung des harmonischen Feldes wäre.

Vielmehr ist, vgl. [22], [3]-[4], [48],

$$
D D x^{i}=\Omega_{l}^{i} x^{l}
$$

mit der Krümmung

$$
\Omega_{l}^{i}=d \omega_{l}^{i}-\omega_{l}^{m} \wedge \omega_{m}^{i} .
$$

Dabei ist das absolute Differential

$$
D \xi^{i}=d \xi^{i}+\omega_{l}^{i} \wedge \xi^{l}
$$

und

$$
\begin{aligned}
D D \xi^{i} & =D\left(d \xi^{i}+\omega_{l}^{i} \wedge \xi^{l}\right) \\
& =d\left(d \xi^{i}+\omega_{l}^{i} \wedge \xi^{l}\right)+\omega_{m}^{i} \wedge\left(d \xi^{m}+\omega_{l}^{m} \wedge \xi^{l}\right) \\
& =\left(d \omega_{l}^{i}+\omega_{m}^{i} \wedge \omega_{l}^{m}\right) \wedge \xi^{l}-\omega_{l}^{i} \wedge d \xi^{l}+\omega_{m}^{i} \wedge d \xi^{m} .
\end{aligned}
$$

Also ist

$$
D D \xi^{i}=\Omega_{l}^{i} \wedge \xi^{l} .
$$

Es gibt - ohne gleich $\Omega_{i}^{l}=0$ anzunehmen - einen Fall, in dem sich eine Differentialgleichung für $\xi$ allein ergibt. Aus

$$
D \xi^{i}=x^{l} \Omega_{l}^{i}
$$

folgt durch Anwendung von $D^{\prime}=-{ }^{\circ} D_{*}$ mit geeignetem * 


$$
D^{\prime} D \xi^{i}=-{ }^{c}\left(\xi^{l} * \Omega_{l}^{i}\right)
$$

falls

$$
D^{\prime} \Omega_{l}^{i}=-{ }^{c} D_{*} \Omega_{l}^{i}=0
$$

vorausgesetzt wird.

Ausser (1.24), d.h. $D^{\prime} \xi_{i}=0$, hat man $D D^{\prime} \xi^{i}=0$ und somit die Differentialgleichung

$$
\left(D^{\prime} D+D D^{\prime}\right) \xi^{i}=\Delta \xi^{i}=-{ }^{c}\left(\xi^{l} \Omega_{l}^{i}\right)
$$

Mittels einer Variationsmethode zeigen wir, dass die Differentialgleichung (2.8) mit der Randbedingung $t_{c} \xi=0$ auf $\partial T$ eine endlichdimensionale Lösungsmannigfaltigkeit $(\xi)=\mathfrak{D}$ hat. Aus dieser schneiden wir dann die Mannigfaltigkeit $\mathscr{D} \cap \mathbb{S}$ der Lösungen heraus, die auch noch die Eigenschaft $D^{\prime} \xi=0$ haben. Es ist schliesslich zu fragen, ob ein so bestimmtes $\xi=D x$ gesetzt werden kann, also wann global ein eventuell auf $T$ mehrdeutiger Vektor $x$ mit $D x=\xi$ gefunden werden kann.

Der Variationsmethode legen wir den Bilinearausdruck

$$
\int{ }_{c} \xi \wedge\left(\Delta \xi+{ }^{c}\left(\xi \wedge{ }_{*} \Omega\right)\right)=\int{ }_{c} \xi \wedge\left(\left(D^{\prime} D \xi+D D^{\prime} \xi\right)+{ }^{c}\left(\xi \wedge_{*} \Omega\right)\right)
$$

mit $D^{\prime}=-{ }^{c} D_{*}$ bzw. $D^{\prime}=-{ }^{*} D_{c}$ zu Grunde, der sich so umformen lässt:

$$
\begin{aligned}
& -\int\left({ }_{c} \xi \wedge\left({ }^{c} D_{*}\right) D \xi+{ }_{c} \xi \wedge D\left(* D_{c}\right) \xi-{ }_{c} \xi \wedge^{c}\left(\xi \wedge_{*} \Omega\right)\right) \\
& =\int\left(D \xi \wedge_{*} D \xi+D_{c} \xi \wedge *_{c} \xi+\xi D_{c} \wedge \xi \wedge_{*} \Omega\right) \\
& =(D \xi \xi, D \xi)+\left(D^{\prime} \xi \xi, D^{\prime} \xi\right)+\left(\xi^{k} \wedge \xi^{l}, \Omega_{k l}\right) \\
& =J(\xi, \xi)
\end{aligned}
$$

Wir erhalten also das Variationsproblem für $\pi={ }_{c} \xi$ mit $t \pi=0$ auf $\partial T$ (2.10) $J(\pi)=(D \pi, D \pi)+\left(D^{\prime} \pi, D^{\prime} \pi\right)-\left({ }^{c} \pi \wedge{ }^{c} \pi, \Omega\right) \rightarrow \min$.

Es kann mit den direkten Methoden behandelt werden unter der vorläufigen Voraussetzung

$$
J(\pi) \geqq K_{1} \sum_{i, \alpha, \beta}\left(\pi_{i, \beta}^{\alpha}\right)^{2}-K_{0} \sum_{i, \alpha}\left(\pi_{i}^{\alpha}\right)^{2},
$$

einer Ungleichheit von der Art der Gårdingschen bzw. der Gaffneyschen:

Am einfachsten folgt so etwas, falls

$$
c_{\alpha \beta}^{i j}=c \delta^{i j} \delta_{\alpha \beta}+\varepsilon_{\alpha \beta}^{i j}, \quad c>0
$$

und $\varepsilon_{\alpha \beta}^{i j}, D \varepsilon$ genügend klein sind. Dann kommt z. B. von der Rotation 
$(D \xi, D \xi)=\left(D^{\prime} \pi, D^{\prime} \pi\right)$

$=\sum_{i} \sum_{(\alpha \gamma)} \pi_{j,[\gamma}^{\beta} c_{\alpha] \beta}^{i j} \pi_{j^{\prime},[\gamma}^{\beta^{\prime}} c_{\alpha] \beta^{\prime}}^{i j^{\prime}}+$ quadratische Glieder in $\pi_{j}^{\beta} \frac{\partial c_{\alpha \beta}^{i j}}{\partial t^{\gamma}}$

$\geqq c^{2} \sum_{i} \sum_{(\alpha, \gamma)}\left(\pi_{i, \gamma}^{\alpha}-\pi_{i, \alpha}^{\gamma}\right)^{2}-\varepsilon \sum_{i} \sum_{\alpha, \beta}\left(\pi_{i, \beta}^{\alpha}\right)^{2}-K \sum_{i} \sum_{\alpha}\left(\pi_{i}^{\alpha}\right)^{2}$.

Analog kommt von der Divergenz

$$
(D \pi, D \pi) \geqq(1-\varepsilon) \sum_{i} \sum_{\alpha}\left(\pi_{i, \alpha}^{\alpha}\right)^{2}-K^{\prime} \sum_{i} \sum_{\alpha}\left(\pi_{i}^{\alpha}\right)^{2} .
$$

Dann folgt nach Friedrichs [14] und Gaffney [15] Ungleichheit (2.11).

Die Frage, für gegebenes $\xi$ die Differentialgleichung

$$
D x^{i}=d x^{i}+\omega_{l}^{i} x^{l}=\xi^{i}
$$

zu lösen, ist von Haahti und Klemola [22] lokal behandelt worden. Dort heisst

$$
D=\psi, \quad x^{i}=f, \quad \omega_{l}^{i}=A, \quad \xi^{i}=a, \quad \Omega_{l}^{i}=R .
$$

Ihre lokale notwendige und hinreichende Bedingung müsste für alle Umgebungen, mit denen die kompakte (geschlossene) Mannigfaltigkeit überdeckt werden kann, gestellt werden:

Bezeichnen wir mit $\mathfrak{X}$ den endlichdimensionalen Raum der Lösungen $\xi$ von $D x=\xi, D^{\prime} \xi=0$, und mit $\mathfrak{A}^{\prime}$ den ebenfalls endlichdimensionalen Raum der konstanten Vektoren $g^{i}$ (der geschlossenen Formen ( $p=0$ )-ten Grades), so muss es einen Teilraum $\mathfrak{X}_{1} \subset \mathfrak{X}$ geben, so dass

$$
(D+\Omega \wedge S \varphi) \mathfrak{X}_{1} \in \Omega \wedge S \mathfrak{H}^{\prime}
$$

gilt mit den Operatoren $S, \varphi$ aus [22].

Ist $\Omega_{i}^{l}=0$, so ist diese Bedingung sicher erfüllt; wegen $D \xi^{i}=\Omega_{l}^{i} x^{i}=0$ reduzieren sich die betrachteten Räume auf den Nullpunkt.

Wir setzen schl:ess'ish die Krümmung

$$
\Omega_{i}^{l}=0
$$

voraus. Das dann aus (1.23), (1.24) entstehende Gleichungssystem ist

$$
\begin{array}{rlrl}
D \xi^{i}=0 & \text { und } \quad D^{\prime} \xi_{i}=0 & \text { in } T \\
n \xi^{i}=0 & \text { d.h. } \quad t \pi_{i}=0 & \text { auf } & \partial T
\end{array}
$$

bei freiem Rand. Dies kommt speziell vor bei einem bewegungsinvarianten Variationsproblem im Euklidischen Raum.

Die Schnittkräfte bei den Selbstspannungen eines mehrfach zusammenhängenden Körpers (oder einer mehrfach zusammenhängenden Schale) in der Nachbarschaft des spannungslosen Zustandes, bzw. die von Volterra 
in seinen Stockholmer »Leçons sur l'intégration des équations différentielles aux dérivées partielles» [74] behandelten sogenannten Distorsionen, bilden linearisiert gerade ein solches verallgemeinertes harmonisches Feld

$$
\begin{gathered}
D v_{h k}=0 \quad \text { und } \quad D^{\prime} v^{h k}=0 \quad \text { in } T, \\
\left(t \pi^{h k}=\right) n v^{h k}=0 \quad \text { auf } \partial T,
\end{gathered}
$$

nur steht jetzt statt des einen Index $i$ der antisymmetrische Doppelindex $h k$ unten.

Hier können die Koordinaten so gewählt werden, dass in $D v$, vgl. (4.8),

$$
\lambda_{0}^{j}=d t^{j}, \quad \lambda_{i}^{j}=0 \text { sonst }
$$

wird. Dann kann man $v_{l k}$ in der Form

$$
v_{l k}=H_{l k}+D X_{l k},
$$

wo $D H=0$ und $d^{\prime} H=0$ in $T, \quad n H=0$ auf $\partial T$ ist, darstellen, und es ergibt sich für $X_{l k}$ ein Variationsproblem

$$
(D X, D X)+2(H, D X) \rightarrow \min ,
$$

das nach den früher von mir [35]-[36] entwickelten Methoden behandelt werden kann. Näheres in Nr. 4, 5.

3. Als zweiter Fall eines verallgemeinerten (pseudo-)harmonischen Feldes seien die normalhyperbolischen Gleichungen der Maxwellschen Theorie für die Ausbreitung des Lichtes im Einsteinschen Gravitationsfeid $g_{\alpha \beta}$ betrachtet. Diesmal ist der indefinite Charakter des Linienelementes einschneidender.

Es handelt sich nur für eine Differentialform um den mehr klassischen Begriff der Elementarlösung (im Sinne Hadamards), also eines Elementardifferentials. Auf dieses, seinen logarithmischen Teil, hat die Struktur des Riemannschen Raumes (durch die Strukturgleichungen) Einfluss und damit auch auf die Art der Abhängigkeit der Lösung von den Anfangswerten: die Frage nach der Gültigkeit des Huygensschen Prinzips im Sinne Hadamards. Wenn Diffusion des Lichtes im Schwerefeld stattfindet, so im Kleinen nur von höherer Ordnung des Verschwindens.

Das elektromagnetische Feld

$$
F=F_{\alpha \beta} d t^{\alpha} d t^{\beta} \quad(\alpha, \beta=1,2,3,4)
$$

im materiefreien Raum ist ein pseudoharmonisches Feld, nämlich eine Lösung der Maxwellschen Gleichungen 
E. HöLdER, Mit harmonischen Feldern verwandte Differentialformen 11

$$
D F=0, \quad D^{\prime} F=0 \text {. }
$$

Hier ist, bezüglich des Riemann-Einsteinschen Linienelementes ${ }^{2}$ )

$$
g_{\alpha \beta} d t^{\alpha} d t^{\beta}=\omega^{4^{2}}-\omega^{1^{2}}-\omega^{2^{2}}-\omega^{3^{2}}
$$

des Schwerefeldes genommen, $D$ das absolute äussere Differential, $D^{\prime}$ das adjungierte absolute äussere Differential.

Das Variationsproblem lautet $\delta I=0$ mit $I=(F, F)$ für $F \rightarrow 0$, d. h. im Kleinen $F=D \varphi$. Damit

$$
0=(F, \delta F)=(F, D \delta \varphi)=\left(D^{\prime} F, \delta \varphi\right) .
$$

Zusammen also $D F=0, \quad D^{\prime} F=0$.

$F$ genügt auch der von Gordon [17] studierten Wellengleichung

$$
\Delta F=\left(D^{\prime} D+D D^{\prime}\right) F=0 \text {, }
$$

ist also eine harmonische Differentialform.

$F$ besitzt als solche eine Integraldarstellung mittels einer harmonischen Elementarlösung

$$
u=\frac{U}{\Gamma}-W \log \Gamma+w
$$

im Sinne Hadamards von $\Delta u=0$. Dabei sind

$$
s^{2}=\Gamma
$$

die geodätische Entfernung des Aufpunktes $\left(t^{i}\right)$ vom Pol $\left(t^{0 i}\right)=(0)$, $U, W, w$ reguläre Differentialformen.

Diese Integraldarstellung für die Lösung des Anfangswertproblems folgt aus der Greenschen Formel (mit Skalarprodukt $(F, G)$ ) für ein 4 -dimensionales Gebiet $T$

$$
\begin{aligned}
& (F, \Delta u)-(u, \Delta F) \\
= & -\int_{\partial T} D^{\prime} F \wedge_{*} u+u \wedge_{*} D F+\int_{\partial T} D^{\prime} u \wedge_{*} F+F \wedge_{*} D u,
\end{aligned}
$$

vgl. Kähler [40].

Nach Analogie von Hadamard [23] oder auch Courant [11] (S. 765 ff.) erkennt man durch Vergleich der logarithmischen Glieder: Das Innere des Abhängigkeitsgebietes $S_{0}$ auf $t=0$ tritt durch einen Term

2) Statt der unabhängigen Variablen $t^{\alpha}$ im ersten Teil steht hier oft $t^{i}=\left(t, t^{i^{\prime}}\right) \quad\left(i=1,2,3,4 ; i^{\prime}=1,2,3\right)$. 


$$
\int_{S_{0}} D^{\prime} W \wedge_{*} F+F \wedge_{*} D W
$$

in Erscheinung, sonst kommen nur Randintegrale der Art

$$
\int_{\partial S_{0}} \cdots
$$

vor.

Huygenssches Prinzip im engeren Sinn Hadamards [23] ("minor premisse») nennt man bei einer Differentialgleichung die Eigenschaft:

Wenn zur Zeit $t=0$ oder exakter in einer kurzen Zeitspanne $-\varepsilon \leqq t \leqq 0$ eine Lichterregung in unmittelbarer Umgebung des Ursprungs $O$ produziert wird, so ist der Effekt zu einer späteren Zeit $t^{*}>0$ in der Nachbarschaft einer Fläche (speziell einer Kugelfläche mit dem Mittelpunkt $O$ und dem Radius $c t^{*}$, also einer dünnen Kugelschale bei der gewöhnlichen Wellengleichung) enthalten. Es herrscht eine schalenförmige Ausbreitung.

Dass auch im Rahmen der allgemeinen Relativitätstheorie der elektromagnetische Wellenvorgang eine scharfe vordere Wellenfront besitzt, hat von Laue [45] eines besonderen Nachweises für wert erachtet - wegen der fundamentalen Bedeutung der Lichtgeschwindigkeit.

Hier handelt es sich aber bei scharf begrenzter Aufgabe eines Lichtsignals um die dauernd scharf begrenzte hintere Front, hinter der es keine Diffusion des Lichtes gibt.

Diese Problematik wird auch auseinander gesetzt von Baker und Copson [1].

Statt des ausstrahlenden Lichtkegels braucht man für die Berechnung des Zustandes im Punkt $O\left(x^{0}\right)$ zur Zeit $t$ aus den Antangswerten im $t^{\prime}$ Raum zur Zeit $t=0$ den von $O, t$ ausgehenden "Antikegel» $\Gamma^{--}$mit der Gleichung

$$
s^{2} \equiv \Gamma=0,
$$

der aus der 3-dimensionalen Anfangsfläche $\left(t^{\prime}\right), \quad t=0$ den Raum $S_{\mathbf{0}}$ ausschneidet.

Das genannte Huygenssche Prinzip im engeren Sinn besagt dann, dass $F(0, t)$ nur von den Anfangswerten

$$
F\left(t^{\prime}, 0\right), \quad \frac{\partial}{\partial t} F\left(t^{\prime}, 0\right)
$$

auf dem Rand $\partial S_{0}=\cap\left(\Gamma^{-}, t=0\right)$ abhängt, nicht von inneren Punkten von $S_{0}$. 
Handelt es sich um ein pseudoharmonisches Feld, so können nur die Anfangswerte der Tangentialteile

$$
t F \quad \text { und } t_{*} F={ }_{*} n F \text { auf } \partial S_{0}
$$

vorgegeben werden. Das Innere von $S_{0}$ kommt nur durch das Glied (3.9) herein.

Wegen (3.9) kommt also beim harmonischen Feld $F$ die Frage der Diffusionsfreiheit, der Gültigkeit des Huygensschen Prinzips, auf das Verschwinden (identisch oder von einer gewissen Ordnung) von $D W$ und $D^{\prime} W$ an.

Bei den pseudoharmonischen Funktionen läuft die Gültigkeit des Huygensschen Prinzips im engeren Sinne Hadamards auf das Verschwinden des logarithmischen Gliedes $W$ selbst hinaus. Die diesbezügliche von Hadamard gestellte Frage ist von Mathisson [54] und später namentlich von P. Günther [18]-[19] gefördert worden. Günther hat für das statische Linienelement das Problem vollständig gelöst.

Die speziell auf das Huygenssche Prinzip bezüglichen (unveröffentlichten) Untersuchungen Stellmachers, die ebenfalls mit Mitteln der (projektiven) Differentialgeometrie das Problem von Hadamard behandeln, haben für uns vor allem insofern Bedeutung, als Stellmacher den Fall in Betracht zieht, dass das Huygenssche Prinzip nur approximativ gilt und von Diffusionsfreiheit verschiedener Ordnung spricht. Sie wurden abgelöst von seiner überraschenden Entdeckung [68]-[69] einer Differentialgleichung 6. Ordnung, die Huygenssch ist und sich nicht auf die Wellengleichung transformieren lässt.

Überhaupt ist die kürzlich (1961 in Oberwolfach, vgl. [11], S. 765 ff.) von Courant mündlich geäusserte ironische Meinung zu bedenken, Hadamards Vermutung (bezüglich des Huygensschen Prinzips) sei ein Musterbeispiel eines nicht korrekt gestellten Problems, vor dem Hadamard sonst mit Recht gewarnt habe. Man solle vielmehr die asymptotische Verteilung in der Nachbarschaft des ausstrahlenden Lichtkegels bei einem scharf aufgegebenen Lichtsignal studieren.

Aber für die elektromagnetischen Wellen hat das Verschwinden der harmonischen Form $W$ selbst keine physikalische Bedeutung. Vielmehr muss man untersuchen, wann die harmonische Form $W$ ein harmonisches Feld, $D W=0, D^{\prime} W=0$ ist.

Hier können wir Stellmacher folgen und wenigstens von Diffusionsfreiheit verschiedener, z. B. niedrigster Ordnung sprechen. Wir können im leeren Einsteinschen Gravitationsfeld die Diffusionsfreiheit wenigstens in 1. Annäherung zeigen. Die aus der Riemannschen Übertragung $\omega_{i j}$ gebildete Krümmungsform 


$$
\Omega_{h k}=d \omega_{h k}-\omega_{h}^{l} \omega_{l k}=\frac{1}{2} R_{h k i j} d t^{i} \wedge d t^{j}
$$

hat hier mit $e_{l} \Omega_{h k}=R_{h k l j} d t^{j}$ die Verjüngung

$$
e^{h} \Omega_{h k}=g^{h l} e_{l} \Omega_{h k}=R_{k j} d t^{j}=0,
$$

und es gilt nach Bianchi bzw. Kähler [40], der den Operator $e_{\boldsymbol{h}}$ benutzt,

$$
D \Omega_{h k}=0, \quad D^{\prime} \Omega_{h k}=0 .
$$

Wir können die schon von Gordon [17] angegebene Form

$$
\Delta u=D^{k} D_{k} u+e_{h} \Omega^{h k} \wedge e_{k} u+\Omega^{h k} e_{h} e_{k} u
$$

entwickeln und die Anfangsfunktion $U$ in (3.6) explizit angeben:

$$
U=\frac{\pi}{\sqrt[4]{|g|}}=\frac{1+c^{i} e_{i}}{\sqrt{1+e_{i} c^{i}}} \pi^{0}
$$

und dann bis zu den Gliedern 3. Grades explizit berechnen. Dazu befolgen wir É. Cartans geometrische Methode des Repère mobile. Dabei ist die Differentialform 2. Grades einer aus $O$ längs der geodätischen Linie parallel verschobenen Plangrösse

$$
\pi=\left(1+c^{i} e_{i}\right) \pi^{0}
$$

mit dem Anfangswert $\pi^{0}$ und ebenso die Differentialform 4. Grades des Volumens

$$
\sqrt{|g|}(d t)=\sqrt{\left|g^{0}\right|} \prod \omega^{i}=\left(1+c_{i}^{i}\right)(d t):
$$

und es ist die Translation $\omega^{i}=d t^{i}+c^{i}$, die ein Repère $R$ in ein differentiell benachbartes $R^{\prime}$ überführt, nach Riemann [12], Vermeil [73], LeviCivita [47], Herglotz [26], Radon [62], am besten aber nach É. Cartan [9] vermöge seiner Strukturgleichungen mittels der Krümmungsdifferentialform $\Omega_{h}{ }^{i}$ und ihrer Ableitungen $\left(\partial_{l} \Omega_{h}{ }^{i}\right)^{0}$ in $O$ ausdrückbar:

$$
c^{i}=\frac{1}{6} t^{h} t^{k}\left(1+\frac{1}{2} t^{l} \partial_{l}\right) e_{k} \Omega_{h}{ }^{i} .
$$

Aus der Entwicklung von $U$ ergibt sich im wesentlichen durch $\Delta U$ das logarithmische Glied $W$ und zwar bis zu den Gliedern 1. Grades. Dass dann wenigstens im Pol $O$ selbst

$$
D W=0 \text { und } D^{\prime} W=0
$$


ist, beruht darauf, dass die Einsteinschen Gravitationsgleichungen (3.12) - auch die kosmologischen - (3.13) nach sich ziehen, und bedeutet, dass die Diffusion des Lichtes im Schwerefeld im Kleinen ein Effekt höherer Ordnung ist.

\section{Ausführung zum bewegungsinvarianten Variationsproblem}

$$
I=\int f(\omega) \rightarrow \min ;
$$

hier hängt das Grundelement

$$
f(\omega)=* f\left(\omega_{\alpha h k}\right)(d t), \quad(d t)=\text { Volumenelement, }
$$

nur ab von der Differentialform der Schraubengeschwindigkeit ("Motor» nach von Mises [56]-[57], insb. S. 196 f.)

$$
\omega_{h k}=\omega_{\alpha h k} d t^{\alpha} \quad\left(h k=\left\{\begin{array}{ll}
01,02,03 & \text { Translationsgeschwindigkeit } \\
23,31,12 & \text { Winkelgeschwindigkeit }
\end{array}\right)\right.
$$

einer (extremalen) Mannigfaltigkeit $e^{0}\left\{R^{0}\left(t^{\alpha}\right)\right\}$ von Triedern, eines »elastischen Körpers» ("Corps déformable») oder einer Schale im Sinne von E. und F. Cosserat [10]; diese wird überdies zum Zwecke der Variation noch in eine (u) -Schar $\left\{R\left(t^{\alpha}, u\right)\right\}$ eingebettet, wobei mit dem alternierenden Tensor $z_{h k}$ der Variation

$$
\bar{\omega}_{h k}=\omega_{h k}+z_{h k} d u
$$

ist. Es gelten die Strukturgleichungen von Cartan [7]-[8]

$$
d \bar{\omega}_{h}^{k}=\bar{\omega}_{h}{ }^{m} \wedge \bar{\omega}_{m}{ }^{\prime}
$$

und zwar auf der Extremalen $e^{0}$ selbst

$$
\Omega_{h}{ }^{k}=d \omega_{h}{ }^{k}-\omega_{h}{ }^{l} \wedge \omega_{l}{ }^{k}=0 .
$$

Die linken Seiten $\Omega$ der in Matrizenform

$$
\left.\Omega=d \omega-\omega \wedge \omega=0 \quad \text { (Koordinaten in } R^{0}\right)
$$

geschriebenen Strukturgleichungen sind Tensoren.

Man zerlegt die Schraubengeschwindigkeit ( = Motor) von $R^{0}(t)$ (gegen ein festes Bezugssystem $\underline{R}$, Koordinaten in $R^{0}$ ) in die Führungsgeschwindigkeit $\lambda$ der Trieder $\left\{R^{1}(t)\right\}$ einer Ausgangsfigur $e^{1}$ plus der Relativgeschwindigkeit $v$ von $R^{0}(t)$ gegen $R^{1}(t)$ (Koordinaten in $R^{0}$ ) 


$$
\omega=\lambda+v .
$$

In

$$
\Omega=(d \lambda-\lambda \wedge \lambda)+(d v-\lambda \wedge v-v \wedge \lambda)-v \wedge v
$$

ist der mittlere Ausdruck

$$
d v-\lambda \wedge v-v \wedge \lambda=D v
$$

das absolute Differential

$$
D v=d t^{\alpha} D_{\alpha} v
$$

mit der absoluten (kovarianten) Ableitung $D_{\alpha}$ in Richtung $t^{\alpha}$. Auch der erste Term

$$
d \lambda-\lambda \wedge \lambda=D \lambda+\lambda \wedge \lambda
$$

hat Tensorcharakter. Wenn man die Koordinaten nachträglich in $R^{1}$ nimmt, sieht man

$$
d \lambda-\lambda \wedge \lambda=0
$$

die Strukturgleichungen für die Ausgangsmannigfaltigkeit $e^{1}$ der Trieder $\left\{R^{1}(t)\right\}$, in deren Nachbarschaft $e^{0}\left\{R^{0}(t)\right\}$ angenommen wird.

Die so gewonnenen Strukturgleichungen

$$
D v=v \wedge v
$$

(alle Koordinaten und Ableitungen genommen in $R^{1}$, womit bei der Bildung von $D$ auch $\lambda$ völlig bekannt ist) sind die erste Gruppe von Differentialgleichungen für das bewegungsinvariante Variationsproblem.

Auf der anderen Seite folgt aus eben diesen Strukturgleichungen für die 1. Variation in der Schar $\bar{\omega}=\omega+z d u$

$$
\left.\begin{array}{l}
\left.\delta \bar{\omega}\right|_{u=0}=\delta \omega=D^{0} z=d z-\omega z+z \omega \\
\delta v=(d z-\lambda z+z \lambda)-v z+z v
\end{array}\right\} \text { (Koordinaten in } R^{0} \text { ). }
$$

Also auch

(4.10) $\delta v=D z-v z+z v \quad$ (Ableitungen und Koordinaten in $R^{1}$ ).

Mit der Entwicklung des Integrationselementes

$$
f(\omega)=f(\lambda)-S(\pi \wedge v)+f^{(2)}(v)
$$

wo die »Dyname» der Schnittkraftformen 2-ten Grades auf $e^{0}$

$$
\pi=f_{\omega}(\lambda)=f_{\lambda}
$$

und $S$ die Spur 


$$
\pi_{l}^{h} \wedge v_{h}^{l}=S(\pi \wedge v)
$$

bedeutet, wird unter Einführung der »Impulsform»

$$
\eta=f_{v}^{(2)}
$$

die erste Variation des Extremalintegrals

$$
\delta I=-\int_{T} S(\pi \wedge \delta v)+S(\eta \wedge \delta v)
$$

nach (4.10)

$$
=-\int_{T} S((\pi+\eta) \wedge(D z-v z+z v))
$$

und in $E^{3}$ wegen

$$
S((\pi+\eta) \cdot z \wedge v)=S(v \wedge(\pi+\eta) \cdot z)
$$

nach Produktintegration

$$
\delta I=\int_{T} S([D(\pi+\eta)+(\pi+\eta) \wedge v-v \wedge(\pi+\eta)] z)-\int_{\partial T} S((\pi+\eta) z) .
$$

Das Verschwinden der ersten Variation des bewegungsinvarianten Variationsproblems gibt also wegen $D \pi=0$ in $T$ und $t \pi=0$ auf $\partial T$ neben

$$
D v=v \wedge v
$$

noch die Euler-Lagrangesche Differentialgleichung für $\eta=f_{v}^{(2)}$

$$
\begin{gathered}
D \eta+\pi \wedge v-v \wedge \pi=-\eta \wedge v+v \wedge \eta \quad \text { in } T \\
t \eta=0 \quad \text { auf } \partial T .
\end{gathered}
$$

Für die spannungslose Ausgangsfigur $e^{1}, \pi=0$, stehen diese Gleichungen linearisiert in Komponentenschreibweise oben, siehe (2.16). Es ist

$$
D^{\prime} v=* D \stackrel{2}{f_{v}}
$$

mit dem quadratischen Anfangsglied $f^{2}$ von $f^{(2)}=\stackrel{2}{f}+f^{(3)}$.

5. Zur Begründung der Zerlegung (2.18) im Euklidischen Raum, auf der dann der Existenzbeweis nach der Variationsmethode beruhen wird, bemerken wir für

$$
D v_{12}=d v_{12}=(v v)_{12}
$$


mit Morreys Potential $\Omega^{+}$, das wir $V^{+}=V$ nennen, die Zerlegung von Kodaira-Morrey [60]

$$
\begin{aligned}
v_{12} & =h_{12}+d X_{12}+d^{\prime} V_{(v v)_{12}} \\
& =H_{12}+D X_{12}+(v v)_{12} .
\end{aligned}
$$

Sodann gibt

$$
D v_{02}=d v_{02}-\lambda_{0}^{l} \wedge v_{2 l}=(v v)_{02}, \quad \lambda_{0}^{l}=-d t^{l},
$$

die Beziehung

$$
d\left(v_{02}-X_{2 l} d t^{l}\right)=h_{2 l} d t^{l}+d^{\prime} V_{(v v)_{2 l}} d t^{l}+(v v)_{02},
$$

somit die Zerlegung

$$
v_{02}+\lambda_{0}^{l} X_{2 l}=h_{02}+d^{\prime} V_{h_{2 l} d l^{l}}+d X_{02}+d^{\prime} V_{(v v)_{02}}+d^{\prime} V_{d^{\prime} V_{(v v)_{2 l} l^{d} l}},
$$

d. h.

$$
\begin{aligned}
v_{02} & =h_{02}+H_{2}+d X_{02}-\lambda_{0}^{l} X_{2 l}+(v v)_{2} \\
& =H_{02}+D X_{02}+(v v)_{2} .
\end{aligned}
$$

So fortfahrend, bekommt man $v_{l k}$ gemäss (2.18) durch die Differentiale $D X_{l k}$ sowie durch harmonische Differentiale und deren Potentiale sowie durch Glieder $(v v)_{2}$ zweiter und höherer Ordnung ausgedrückt.

Hierbei gehören die zunächst auftretenden harmonischen Differentialformen $h_{l k} \mathrm{zu}$ Morreys Raum ([60], Theorem 5.5)

$$
\mathfrak{H}^{+}: \quad n h_{l k}=0 \text { auf } \partial T .
$$

Wir setzen hier noch voraus, dass die Form $(\xi, \xi)$ positiv definit ist:

$$
(\xi, \xi)=\int c^{h k, \alpha ; l m, \beta} \xi_{h k, \alpha} \xi_{l m, \beta}(d t) \geqq C \int \sum\left(\xi_{h k, \alpha}\right)^{2}(d t) .
$$

Es ist danı das Diricmetmtegraı

$$
(D X, D X)=0
$$

nur für $D X=\xi=0$. Diese zu sich selbst parallel verschobenen (»konstanten») Tensoren $\left(X_{h k}\right)$ bedeuten feste (infinitesimale) Verschraubungen $X$ des starren elastischen Körpers und bilden einen 6-dimensionalen Raum $\mathfrak{A}$.

Diese Voraussetzung (5.4) ersetzt die Gårdingsche Ungleichung, und es folgt wie in meiner Arbeit [35] (S. 45, (5.5)) die Ungleichheit

$$
(D X, D X) \geqq K_{1}((X, X))-L_{1}(X, X),
$$




$$
\begin{aligned}
((X, X)) & =(X, X)+\sum_{q} \int_{G_{q}} \sum_{(h k), \alpha}\left(X_{h k, t \alpha}\right)^{2} d t, \\
(X, X) & =\sum_{q} \int_{G_{q}} \sum_{(h k)}\left(X_{h k}\right)^{2} d t .
\end{aligned}
$$

Für jede von $X=$ const verschiedene wirkliche Deformation $X \perp \mathfrak{\Re}$ gilt eine Abschätzung

$$
(\xi, \xi)=(D X, D X) \geqq \lambda_{0}((X, X)) .
$$

Für $X$ stellen wir das Variationsproblem

$$
I(X)=(D X, D X)+2(H, D X) \rightarrow \min , \quad X \in \mathfrak{P}_{2}^{+},
$$

d. h. für $\xi=D X$

$$
I=(\xi, \xi)+2(H, \xi) \rightarrow \min .
$$

Wegen

$$
2|\alpha \beta| \leqq \eta \alpha^{2}+\eta^{-1} \beta^{2} \quad \text { mit jedem } \quad \eta>0
$$

gilt

$$
I \geqq(1-\eta)(\xi, \xi)-\frac{1}{\eta}(H, H) .
$$

Der abgezogene Term

$$
\frac{1}{\eta}(H, H)
$$

ist fest. Ersetzt man $\eta$ durch $\lambda_{0} / 2$, so folgt

$$
I \geqq \frac{\lambda_{0}}{2}((X, X))-\frac{2}{\lambda_{0}}(H, H)
$$

für alle $\mathfrak{P}_{2}^{+}$-Vektoren $X \perp \Re$. Die $I$-Werte sind zufolge nach unten beschränkt.

In jeder Minimalfolge $\left\{X_{n}\right\}$ ist $((X, X))$ gleichmässig beschränkt. Also existiert eine schwach konvergente Teilfolge.

Damit ist die Existenz des minimisierenden Vektors $X \perp \mathfrak{\Re}$ für das unterhalbstetige $I(X)$ bewiesen.

Ist $X$ der minimisierende Vektor und $z \in \mathfrak{P}_{2}^{+}$eine Variation, so muss

$$
\begin{aligned}
I(X) & \leqq I(X+\lambda z) \\
& =I(X)+2 \lambda((D X, D z)+(H, D z))+\lambda^{2}(D z, D z)
\end{aligned}
$$

sein, d. h. 
(5.13a)

$$
(D X, D z)+(H, D z)=0,
$$

und cum grano salis:

$$
\left(D^{c} D X, z\right)+\left(D^{c} H, z\right)=0 .
$$

Also

$$
\begin{aligned}
D^{c}(D X+H) & =0, \\
t^{c}(D X+H) & =0,
\end{aligned}
$$

d. h. für $v=H+D X$ gilt ausser $D v=0$

$$
D^{\prime} v=0 \quad \text { in } T, \quad t^{c} v=n v=0 \quad \text { auf } \partial T,
$$

wie es sein sollte.

Beim nichtlinearen bewegungsinvarianten Variationsproblem im euklidischen Raum löst man die Darstellungsformel (5.2) auf:

$$
v=I I+D X+(H, D X)_{2}
$$

mit einem Funktional $(H, D X)_{2}$ vom zweiten und höheren Grad.

Nun gibt die Anwendung von $D^{\prime}$ auf Grund der Gleichgewichtsbedingungen (4.17) bei fehlenden Vorspannungen $\pi$

$$
D^{\prime} D X=\Delta X=-D^{\prime} H-D^{\prime}(H, D X)_{2}+D^{\prime} v=q\{H \mid D X\},
$$

wo rechts in den Gliedern höherer Ordnung ebenfalls für $v$ der Ausdruck (5.16) einzusetzen ist,

$$
D^{\prime} v=*\left[v \wedge f_{v}^{(2)}-f_{v}^{(2)} \wedge v\right]-* D f_{v}^{(3)} .
$$

Diese Gleichung lässt sich durch sukzessive Approximationen auflösen, falls man bei freien Randwerten den Green-de Rhamschen Operator im Sinne meiner Arbeit [35]-[36] besitzt.

Johannes Gutenberg -Universität

Mainz, Deutschland 


\section{Literatur}

[1] Baker, B. B., und E. T. Copson: The mathematical theory of Huygens' principle. - 2. Aufl., Clarendon Press, Oxford, 1950.

[2] Biezeno, C. B., und R. Grammel: Technische Dynamik. - Verlag von Julius Springer, Berlin, 1939.

[3] Bochner, S.: A new viewpoint in differential geometry. - Canad. J. Math. 3, 1951 , S. $460-470$.

[4] - - Curvature and Betti numbers in real and complex vector bundles. - Univ. e Politec. Torino. Rend. Sem. Mat. 15, 1955/1956, S. 225-253.

[5j Boerner, H.: Über die Legendresche Bedingung und die Feldtheorien in der Variationsrechnung der mehrfachen Integrale. - Math. Z. 46, 1940, S. $720-742$.

[6] Cartan, É.: Sur les variétés à connexion affine et la théorie de la relativité généralisée. I. - Ann. Sci. École Norm. Sup. (3) 40, 1923, S. 325-412. = Oeuvres complètes. III. 1, Gauthier-Villars, Paris, 1955, S. 659-746.

[7] -»- Leçons sur la théorie des espaces à connexion projective. - Cahiers Scientifiques XVII, Gauthier-Villars, Paris, 1937.

[8] — - La théorie des groupes finis et continus et la géométrie différentielle traitées par la méthode du repère mobile. - Cahiers Scientifiques XVIII, Gauthier-Villars, Paris, 1937.

[9] -»- Leçons sur la géométrie des espaces de Riemann. - 2. Aufl., Cahiers Scientifiques II, Gauthier-Villars, Paris, 1951.

[10] Cosserat, E. und F.: Théorie des corps déformables. - Librairie Scientifique A. Herniann et Fils, Paris, 1909.

[11] Courant, R., und D. Hilbert: Methods of mathematical physics. II. Partial differential equations. - Interscience Publishers, NewYork/London, 1962.

[12] Dedekind, R., und H. Weber: Anmerkungen (zu der Abhandlung »Ueber die Hypothesen, welche der Geometrie zu Grunde liegen» von B. RiEmanN und zu seiner Beantwortung einer von der Pariser Akademie gestellten Preisaufgabe). - Bernhard Riemann's gesammelte mathematische Werke und wissenschaftlicher Nachlass, 2. Aufl., Druck und Verlag von B. G. Teubner, Leipzig, 1892, S. 405-423.

[13] Duff, G. F. D.: Harmonic $p$-tensors on normal hyperbolic Riemannian spaces. Canad. J. Math. 5, 1953, S. 57-80.

[14] Friedrichs, K. O.: Differential forms on Riemannian manifolds. - Comm. Pure Appl. Math. 8, 1955, S. 551-590.

[15] Gaffney, M. P.: Hilbert space methods in the theory of harmonic integrals. Trans. Amer. Math. Soc. 78, 1955, S. 426-444.

[16] Gånding, L.: Dirichlet's problem for linear elliptic partial differential equations. - Math. Scand. 1, 1953, S. 55-72.

[17] Gordon, W.: Zur Lichtfortpflanzung nach der Relativitätstheorie. - Ann. Physik (IV) 72,1923 , S. $421-456$. 
[18] GüNTher, P.: Zur Gültigkeit des Huygensschen Prinzips bei partiellen Differentialgleichungen vom normalen hyperbolischen Typus. - Ber. Verh. Sächs. Akad. Wiss. Leipzig. Math.-Nat. Kl. 100:2, 1952.

[19] -»- Über einige spezielle Probleme aus der Theorie der linearen partiellen Differentialgleichungen zweiter Ordnung. - Ber. Verh. Sächs. Akad. Wiss. Leipzig. Math.-Nat. Kl. 102:1, 1957.

[20] GüNTHER, W.: Über das Gleichgewicht an einer randbelasteten Schale. - Abh. Braunschweig. Wiss. Ges. 8, 1956, S. 111-120.

[21] -»- Zur Statik und Kinematik des Cosseratschen Kontinuums. - Abh. Braunschweig. Wiss. Ges. 10, 1958, S. 195-213.

[22] Налнті, H., und T. Klemola: Zur Theorie der vektorwertigen Differentialformen. - Ann. Acad. Scient. Fennicæ A. I. 318, 1962.

[23] Hadamard, J.: Le problème de Cauchy et les équations aux dérivées partielles linéaires hyperboliques. - Hermann \& $\mathrm{C}^{\text {ie }}$, Paris, 1932.

[24] -»- The problem of diffusion of waves. - Ann. of Math. 43, 1942, S. 510-522.

[25] Herglotz, G.: Zur Riemannschen Metrik. - Ber. Verh. Sächs. Akad. Wiss. Leipzig. Math.-Nat. Kl. 73, 1921, S. 215-225.

[26] -»- Über die Bestimmung eines Linienelementes in Normalkoordinaten aus dem Riemannschen Krümmungstensor. - Math. Ann. 93, 1925, S. 46-53.

[27] Hestenes, M. R.: Applications of the theory of quadratic forms in Hilbert space to the calculus of variations. - Pacific J. Math. 1, 1951, S. 525-581.

[28] -»- Quadratic variational theory and linear elliptic partial differential equations. - Trans. Amer. Math. Soc. 101, 1961, S. 306-350.

[29] Hildebrandt, S.: Über die Identität der Sobolewschen und der CalkinMorreyschen Räume. - Math. Ann. 148, 1962, S. 226-237.

[30] - - Rand- und Eigenwertaufgaben bei stark elliptischen Systemen linearer Differentialgleichungen. - Math. Ann. 148, 1962, S. 411-429.

[31] Hodge, W. V. D.: The theory and applications of harmonic integrals. - 2. Aufl., Cambridge University Press, Cambridge, 1952.

[32] Hölder, E.: Über die explizite Form der dynamischen Gleichungen für die Bewegung eines starren Körpers relativ zu einem geführten Bezugssystem.

- Z. Angew. Math. Mech. 19, 1939, S. 166-176.

[33] -»- Das Eigenwertkriterium der Variationsrechnung zweifacher Extremalintegrale. - Bericht über die Mathematiker-Tagung in Berlin, Januar 1953, Deutscher Verlag der Wissenschaften, Berlin, 1953, S. 291-302.

[34] -»- Die Dynamik des starren Körpers in einem nichteuklidischen Raum. Abh. Math. Sem. Univ. Hamburg 20, 1956, S. 242-252.

[35] - - Über die partiellen Differentialgleichungssysteme der mehrdimensionalen Variationsrechnung. - Jber. Deutsch. Math. Verein. 62, 1959, S. 34-52.

[36] -»- Beweise einiger Ergebnisse aus der Theorie der 2. Variation mehrfacher Extremalintegrale. - Math. Ann. 148, 1962, S. 214-225.

[37] van Hove, L.: Sur l'extension de la condition de Legendre du calcul des variations aux intégrales multiples à plusieurs fonctions inconnues. - Nederl. Akad. Wetensch. Proc. 50, 1947, S. 18-23. = Indagationes Math. 9, 1947, S. 3-8.

[38] - - - Sur le signe de la variation seconde des intégrales multiples à plusieurs fonctions inconnues. - Acad. Roy. Belg. Cl. Sci. Mém. Coll. in $8^{\circ}$ (2) 24:5, 1949.

[39] KäHLER, E.: Bemerkungen über die Maxwellschen Gleichungen. - Abh. Math. Sem. Hansisch. Univ. 12, 1936, S. 1-28. 
[40] KäHLER, E.: Innerer und äusserer Differentialkalkül. - Abh. Deutsch. Akad. Wiss. Berlin. Kl. Math. Phys. Tech. 1960:4.

[41] -»- Die Dirac-Gleichung. - Abh. Deutsch. Akad. Wiss. Berlin. Kl. Math. Phys. Tech. 1961:1.

[42] Klötzler, R.: Bemerkungen zu einigen Untersuchungen von M. I. Višik im Hinblick auf die Variationsrechnung mehrfacher Integrale. - Math. Nachr. $17,1958 / 1959$, S. $47-56$.

[43] Kodaira, K.: Harmonic fields in Riemannian manifolds (Generalized potential theory). - Ann. of Math. (2) 50, 1949, S. 587-665.

[44] von Laue, M.: Die Relativitätstheorie. II. Die allgemeine Relativitätstheorie und Einsteins Lehre von Schwerkraft. - Wissenschaft 68, Friedr. Vieweg \& Sohn, Braunschweig, 1921.

[45] -»- Die Bedeutung des Nullkegels in der allgemeinen Relativitätstheorie. S.-B. Preuss. Akad. Wiss. Math.-Phys. Kl. 1922, S. 118-126.

[46] De La Vallée Poussin, Ch. J.: Leçons de mécanique analytique. I. - 2. Aufl., Librairie Universitaire / Gauthier-Villars, Louvain/Paris, 1932.

[47] Levi-Civita, T.: Sur l'écart géodésique. - Math. Ann. 97, 1927, S. 291-320.

[48] Lichnerowicz, A.: Théorie globale des connexions et des groupes d'holonomie. Consiglio Nazionale delle Ricerche. Monografie Matematiche 2, Edizioni Cremonese, Roma, 1955.

[49] -»- Théories relativistes de la gravitation et de l'électromagnétisme. Relativité générale et théories unitaires. - Collection d'Ouvrages de Mathématiques à l'Usage des Physiciens, Masson et $\mathrm{C}^{\mathrm{ie}}$, Paris, 1955.

[50] Lichtenstein, L.: Untersuchungen über zweidimensionale reguläre Variationsprobleme. I. Das einfachste Problem bei fester Begrenzung. Jacobische Bedingung und die Existenz des Feldes. Verzweigung der Extremalflächen. - Monatsh. Math. Phys. 28, 1917, S. 3-51.

[51] -》- Untersuchungen über zweidimensionale reguläre Variationsprobleme. II. Das einfachste Problem bei fester und bei freier Begrenzung. - Math. Z. 5, 1919, S. $26-51$.

[52] LounivaAra, I. S.: Über das Dirichletsche Problem für die selbstadjungierten linearen partiellen Differentialgleichungen zweiter Ordnung. - Rend. Circ. Mat. Palermo (2) 5, 1956, S. 260-274.

[53] -»- Zur Theorie der Unterräume in linearen Räumen mit indefiniter Metrik. Ann. Acad. Scient. Fennicæ A. I. 252, 1958.

[54] Mathisson, M.: Le problème de M. Hadamard relatif à la diffusion des ondes. Acta Math. 71, 1939, S. 249-282.

[55] Maurin, K.: Elliptizität und schwache Halbstetigkeit gewisser Funktionale der Variationsrechnung mehrfacher Integrale. Vollstetigkeit Greenscher Transformationen. - Studia Math. 17, 1958, S. 175-187.

[56] von Mises, R.: Motorrechnung, ein neues Hilfsmittel der Mechanik. - Z. Angew. Math. Mech. 4, 1924, S. 155-181.

[57] -»- Anwendungen der Motorrechnung. - Z. Angew. Math. Mech. 4, 1924, S. $192-213$.

[58] Morrey, C. B., Jr.: Second order elliptic systems of differential equations. Contributions to the theory of partial differential equations, Annals of Mathematics Studies 33, Princeton University Press, Princeton, 1954, S. $101-159$.

[59] -》- A variational method in the theory of harmonic integrals. - Comm. Pure Appl. Math. 9, 1956, S. 499-508. 
[60] Morrey, C. B., JR.: A variational method in the theory of harmonic integrals, II. Amer. J. Math. 78, 1956, S. 137-170.

[61] Nevanlinna, R.: Über metrische lineare Räume. I. Allgemeine Bemerkungen zur Metrisierbarkeit. II. Bilinearformen und Stetigkeit. III. Theorie der Orthogonalsysteme. IV. Zur Theorie der Unterräume. V. Relationen zwischen verschiedenen Metriken. - Ann. Acad. Scient. Fennicæ A. I. 108/113/115/163/222, 1952/1952/1952/1954/1956.

[62] Radon, J.: Bestimmung einer Riemannschen Metrik durch Krümmungseigenschaften. - Monatsh. Math. Phys. 35, 1927, S. 9-24.

[63] Rellich, F.: Ein Satz über mittlere Konvergenz. - Nachr. Ges. Wiss. Göttingen. Math.-Phys. Kl. 1930, S. 30-35.

[64] De Rham, G.: Variétés différentiables. Formes, courants, formes harmoniques. Actualités Sci. Ind. 1222, Hermann \& $C^{\text {ie }}$, Paris, 1955.

[65] Riesz, M.: L'intégrale de Riemann-Liouville et le problème de Cauchy. - Acta Math. 81, 1949, S. 1-223.

[66] Schoutex, J. A.: Ricci-calculus. An introduction to tensor analysis and its geometrical applications. - 2. Aufl., Grundlehren der mathematischen Wissenschaften X, Springer-Verlag, Berlin/Göttingen/Heidelberg, 1954.

[67] Schwarz, H. A.: Ueber ein die Flächen kleinsten Flächeninhalts betreffendes Problem der Variationsrechnung. Festschrift zum Jubelgeburtstag des Herrn Karl Weierstrass. - Acta Soc. Scient. Fennicæ 15, 1888, S. 315-362. $=$ Gesammelte mathematische Abhandlungen. I, Verlag von Julius Springer, Berlin, 1890, S. 223-269.

[68] Stellmacher, K. L.: Ein Beispiel einer Huyghensschen Differentialgleichung. Nachr. Akad. Wiss. Göttingen. Math.-Phys. Kl. II. a. 1953, S. $133-138$.

[69] -»- Eine Klasse huyghenscher Differentialgleichungen und ihre Integration. Math. Ann. 130, 1955, S. 219-233.

[70] Study, E.: Geometrie der Dynamen. Die Zusammensetzung von Kräften und verwandte Gegenstände der Geometrie. - Druck und Verlag von B. G. Teubner, Leipzig, 1903.

[71] -»- Sur les équations du mouvement d'un corps solide. - J. Math. Pures Appl. (6) 7,1911, S. $97-111$.

[72] Treffrz, E.: Ableitung der Schalenbiegungsgleichungen mit dem Gastiglianoschen Prinzip. - Z. Angew. Math. Mech. 15, 1935, S. 101-108.

[73] Vermeil, H.: Bestimmung einer quadratischen Differentialform aus der Riemannschen und den Christoffelschen Differentialinvarianten mit Hilfe von Normalkoordinaten. - Math. Ann. 79, 1918, S. 289-312.

[74] Volterra, V.: Leçons sur l'intégration des equations différentielles aux dérivées partielles, professées à Stockholm. - Almqvist \& Wiksell, Uppsala, 1906.

[75] WEYL, H.: Raum, Zeit, Materie. Vorlesungen über allgemeine Relativitätstheorie. - 5. Aufl., Verlag von Julius Springer, Berlin, 1923.

Gedruckt Januar 1964. 